\title{
Immunotherapeutic Approaches to Target Cancer Stem Cell: Progress and Challenges
}

Sachin Kumar Deshmukh*

Department of Oncologic Sciences, Mitchell Cancer Institute, University of South Alabama, AL 36604, USA

\section{Editorial}

Immunotherapy referred to biological therapy that uses the potential of individual's own immune system to fight cancer. Immunotherapeutic approaches include the use of cytokines, chemokines, monoclonal antibodies, cancer vaccines and immune cells. Immunotherapy is considered one of the most promising and exciting areas for the treatment of cancer.

The success of immunotherapeutic approaches for melanoma and non-small cell lung cancer raised hope for the treatment of other malignancies as well. However, resistance to the cancer therapies developed by tumor cells is the biggest challenge in the cancer treatment. Due to the heterogeneous nature of cancer, the bulk tumor consists of diverse cells having distinct molecular, morphological and phenotypic signatures with differential levels of sensitivity to treatment $[1,2]$. The cancer stem cells (CSCs) that are rare immortal cells of bulk tumor have the capability to self-renew by dividing and give rise to many cell types that constitute the tumor. CSCs are increasingly identified as the source of tumor progression, metastasis, and cancer therapy resistance and relapse $[2,3]$. Treatment strategies that can eliminate CSCs and nonCSCs suggested improving the long-term clinical outcome for cancer patients. Since immunotherapy directly utilizes the immune cells and works in the distinct principle from chemotherapy or small molecule therapy, it can be an alternative therapeutic approach to target CSCs.

Identification and validation of target specific to CSCs are crucial for successful execution of immunotherapy. EGFRvIII, a truncated version of EGFR expressed in glioblastoma (GBM) CSCs suggested being a good target for immune interventions [4]. EGFRvIII variants are expressed across the various other tumor types and their expression is often correlated with poor clinical outcome. Peptide CDX-110, derived from EGFRvIII showed high immunogenicity to anti-EGFRvIII monoclonal antibodies (MAbs). Moreover, EGFRvIII-specific dendritic cell vaccine and CDX-110-KLH peptide vaccine demonstrated potential in preclinical and clinical studies for GBM treatment [5]. In addition, chimeric antigen receptor (CAR) T cell redirected to the EGFRvIII is in the process of development for the treatment of GBM patients [6] Acute Myeloid Leukemia (AML) CSCs expressed Cyclin A1 and WT-1 is recognized as the target of the immunotherapeutic approach. T cell targeting Cyclin A1 and WT-1 are generated and expected to be tested in preclinical models soon [7]. Natural killer cell (NK) immunotherapy is the other immune cell-based therapy that has been explored to target CSCs. Colorectal cancer CSCs demonstrated susceptibility to NK killing due to the upregulation of the ligands for the activating natural cytotoxicity receptors NKp30 and NKp44 [8]. The breast cancer CSCs showed sensitivity to IL-15 and IL-2 activated NK cells. The activity of NK cells was suggested to mediate by elevated expression of the NKG2D ligands ULBP1, ULBP2, and MICA on breast CSCs [9]. Further, melanoma CSCs showed sensitivity to IL-2 activated NK cell cytotoxicity which suggested to mediated by the DNAM-1 ligands Nestin-2 and PVR [10].

The tumor microenvironment (TME) which consists of cancer cells, CSCs, fibroblast, resident and recruited immune cells is a critical barrier to overcome in order to target CSCs. The efficacy of immunotherapeutic can be improved by targeting specific and appropriate antigen, inducing superior immune cell response and facilitating the infiltration of tumorreactive $\mathrm{T}$ cells and NK cells within the TME. Efficacy of $\mathrm{T}$ cell can be improved by substituting amino acid residues within the complementary determining region 3 (CDR3) of the $\mathrm{T}$ cell receptor (TCR) chains for avoiding the possibilities of toxicities due to cross-reactivity against unintended targets. Similarly, NK cell immunotherapeutic approaches can be improved by promoting NK cells longevity in vivo, increasing the homing of NK cells to tumor sites and inducing hyperresponsiveness. Other strategies that can enhance the effectiveness of immunotherapy using a combination approach by integrating monoclonal antibody, cytokines, vaccines, T cells and NK cells therapy. As our understanding of immunotherapy and CSCs biology improved in recent years, certainly this will lead us to target CSCs with better therapeutic strategies to improve the clinical outcomes for cancer patients.

\section{References}

1. Farkona S, Diamandis EP, Blasutig IM (2016) Cancer immunotherapy: The beginning of the end of cancer? BMC Med 14: 73.

2. Dagogo-Jack I, Shaw AT (2018) Tumor heterogeneity and resistance to cancer therapies. Nat Rev Clin Oncol 15: 81-94.

3. Deshmukh SK (2017) Breast cancer stem cells: Understanding and opportunities for therapeutics. J Stem Cell Regen Biol 3: 163-164.

4. Emlet DR, Gupta P, Holgado-Madruga M, Del Vecchio CA Mitra SS, et al (2014) Targeting a glioblastoma cancer stem-cell population defined by EGF receptor variant III. Cancer Res 74: 1238-1249.

5. Chistiakov DA, Chekhonin IV, Chekhonin VP (2017) The EGFR variant III mutant as a target for immunotherapy of glioblastoma multiforme. Eur J Pharmacol. 810: $70-82$

6. O'Rourke DM, Nasrallah MP, Desai A, Melenhorst JJ, Mansfield K, et al (2017) A single dose of peripherally infused EGFRVIII-directed CAR T cells mediates antigen loss and induces adaptive resistance in patients with recurrent glioblastoma. Sci Transl Med 9: eaaa0984.

7. Ochsenreither S, Majeti R, Schmitt T, Stirewalt D, Keilholz U, et al. (2012) Cyclin-A1 represents a new immunogenic targetable antigen expressed in acute myeloid leukemia stem cells with characteristics of a cancer-testis antigen. Blood 119: 5492-5501.

8. Tallerico R, Todaro M, Di Franco S, Maccalli C, Garofalo C, et al. (2013) Human NK cells selective targeting of colon cancer-initiating cells: A role for natural cytotoxicity receptors and MHC class I molecules. J Immunol 190: 2381-2390.

9. Yin T, Wang G, He S, Liu Q, Sun J, et al. (2016) Human cancer cells with stem cell-like phenotype exhibit enhanced sensitivity to the cytotoxicity of IL-2 and IL-15 activated natural killer cells. Cell Immunol 300: 41-45.

10. Pietra G, Manzini C, Vitale M, Balsamo M, Ognio E, et al. (2009) Natural killer cells kill human melanoma cells with characteristics of cancer stem cells. Int Immunol 21: 793-801.

*Corresponding author: Sachin Kumar Deshmukh, Department of Oncologic Sciences, Mitchell Cancer Institute, University of South Alabama, 1660 SpringHill Avenue, Mobile, AL 36604-1405, USA Tel: 251-445-9872;E-mail:skdeshmukh@health.southalabama.edu

Received July 09, 2018; Accepted July 10, 2018; Published July 12, 2018

Citation: Deshmukh SK (2018) Immunotherapeutic Approaches to Target Cancer Stem Cell: Progress and Challenges. J Cancer Sci Ther 10: 151-151. doi: 10.4172/1948-5956.1000e136

Copyright: (C) 2018 Deshmukh SK. This is an open-access article distributed under the terms of the Creative Commons Attribution License, which permits unrestricted use, distribution, and reproduction in any medium, provided the original author and source are credited. 